\title{
Interference-temperature limit for cognitive radio networks with MIMO primary users
}

\author{
C. Lameiro*, W. Utschick ${ }^{\dagger}$ and I. Santamaría* \\ *Dept. of Communications Engineering, Universidad de Cantabria, Spain \\ Email: \{lameiro,nacho\}@gtas.dicom.unican.es \\ ${ }^{\dagger}$ Associate Institute for Signal Processing, Technische Universität München, Germany \\ Email: utschick@tum.de
}

\begin{abstract}
This paper derives the interference-temperature (IT) limit for a multi-antenna primary user (PU) with a rate constraint. While in the case of a single-antenna PU there is a one-to-one mapping between IT and achievable rate, this correspondence does not hold anymore when a multiple-input multiple-output (MIMO) system is considered. In such cases, the spatial distribution of the interference must be taken into account, since it strongly affects the PU performance. To this end, we derive a closed-form expression for the maximum IT that can be tolerated by identifying the worst-case interference covariance matrix, which results in a multilevel waterfilling problem.

Index Terms-Interference temperature, cognitive radio.
\end{abstract}

\section{INTRODUCTION}

Future wireless communication networks are envisioned to be comprised of heterogeneous networks (HetNets), in order to provide sufficiently high data rates to the ever-increasing number of wireless devices, and, at the same time, reducing the cost and increasing their flexibility. Such scenarios, however, are mainly limited by interference, and hence the design of suitable interference management techniques becomes crucial to make an efficient use of the scarce radio resources. In this context, underlay cognitive radio (CR) has been proposed [1] [3], so that the so-called secondary users (SU) are allowed to coexist with the legacy or primary network as long as a minimum performance at the latter is guaranteed. To this end, interference temperature (IT) is typically adopted as a means of measure and constrain the impact of the SUs on the legacy network. Following this approach, the interference from the SUs must be below a given threshold, which is selected such that a satisfactory performance of the primary users (PUs) is guaranteed.

Network coexistence in the context of underlay CR has been widely studied in the literature (see, e.g., [4]-[9] and references therein). All these works, however, focus solely on optimizing the performance of the SUs, and assume the IT constraints to be known a priori. Theoretically, the IT is directly related to the capacity by the Shannon formula [10]

$$
C=W \log _{2}\left(1+\frac{S}{I}\right),
$$

where $W$ is the channel bandwidth, $S$ the signal power and $I$ the interference plus noise power. Thus, in order to sustain a given data rate, the maximum IT that can be tolerated is univocally given by the foregoing expression. However, when a multiple-input multiple-output (MIMO) PU is considered, this is no longer the case since the total interference level cannot be uniquely determined from the rate. The problem of obtaining IT thresholds for a MIMO PU was already considered in [11], where a suboptimal value was derived. This IT level was used in [11] merely as a means to assess the performance of different techniques with respect to the IT approach. However, if the optimal IT value is not used for such comparisons, the results obtained this way may be misleading.

In this work, we consider the problem of obtaining the maximum IT threshold for a MIMO PU that has a rate constraint. First, we show that the problem is equivalent to identifying the worst-case interference covariance matrix, i.e., the one with the most detrimental spatial signature. Then, the structured of the worst spatial signature is obtained, showing that the interference power at each direction admits a multilevel waterfilling solution.

Notation: Bold upper case letters denote matrices, and lightface letters denote scalar quantities. We use $(\mathbf{A})^{H},|\mathbf{A}|$ and $\operatorname{Tr}(\mathbf{A})$ to denote Hermitian, determinant and trace operation, respectively. $\mathcal{C N}\left(0, \sigma^{2}\right)$ stands for circularly-symmetric complex Gaussian random variable with zero mean variance $\sigma^{2} . \mathbb{C}^{M \times N}$ is the space of $M \times N$ complex matrices, and $\mathbb{S}_{+}^{N}$ denotes the set of $N \times N$ positive semidefinite matrices. For squared Hermitian matrices $\mathbf{A}$ and $\mathbf{B}, \mathbf{A} \succeq \mathbf{B}$ means that $\mathbf{A}-\mathbf{B}$ is positive semidefinite. The operation $(a)^{+}$ means $\max (a, 0)$. We use $\mathbf{I}$ to denote an identity matrix of the appropriate dimensions. The optimal solution of an optimization problem is indicated by $(\cdot)^{\star}$.

\section{INTERFERENCE-TEMPERATURE LIMIT}

Let us consider a primary point-to-point link with $M$ transmit antennas and $N$ receive antennas. Denoting the MIMO channel by $\mathbf{H} \in \mathbb{C}^{N \times M}$ and the transmit covariance matrix by $\mathbf{Q} \in \mathbb{S}_{+}^{M \times M}$, the achievable rate of this primary link in the absence of interference is given by

$$
R=\log \left|\mathbf{I}+\frac{1}{\sigma^{2}} \mathbf{H Q H} \mathbf{H}^{H}\right|,
$$

where $\sigma^{2}$ is the variance of the additive white Gaussian noise (AWGN) at the receiver. Since the PU has a rate constraint, 
the following must hold

$$
R(\mathbf{K})=\log \left|\mathbf{I}+\left(\sigma^{2} \mathbf{I}+\mathbf{K}\right)^{-1} \mathbf{H Q H}^{H}\right| \geq \bar{R},
$$

where $\bar{R}$ is the rate requirement for the primary link and $\mathbf{K} \in \mathbb{S}_{+}^{N \times N}$ is the interference covariance matrix. According to the IT framework, the total interference power at the primary receiver is constrained to be below a given threshold, i.e., $\operatorname{Tr}(\mathbf{K}) \leq t$, where $t$ is selected such that (3) holds for all interference covariance matrices satisfying the IT constraint. However, while in the single-antenna case there is a one-toone mapping between IT and rate, this property does not hold anymore when the PU is equipped with multiple antennas. In this case, the spatial structure of the interference comes up, strongly affecting the achievable rate of the primary link. Cumanan et al. proposed in [11] a simple expression for selecting an IT threshold to ensure (3), by bounding the maximum rate reduction due to the interference. This threshold is obtained as

$$
\tilde{t}=2^{\frac{1}{\min (M, N)}\left(\log _{2}\left|\sigma^{2} \mathbf{I}+\mathbf{H Q} \mathbf{H}^{H}\right|-\bar{R}\right)}-\sigma^{2} .
$$

Eq. (4), however, provides a too conservative IT threshold, which is not the largest possible value. Since we are concerned with how to compute the optimal threshold, we first express this problem in its most general form as follows.

$$
\begin{array}{lll}
\mathcal{P}_{1}: & \underset{t}{\operatorname{maximize}} & t, \\
& \text { subject to } & R(\mathbf{K}) \geq \bar{R}, \forall \mathbf{K} \in \mathcal{K}_{t},
\end{array}
$$

where the set $\mathcal{K}_{t}$ is defined as

$$
\mathcal{K}_{t}=\{\mathbf{K} \succeq 0: \operatorname{Tr}(\mathbf{K}) \leq t\}
$$

In the foregoing problem, the rate requirement must be satisfied for all interference covariance matrices in $\mathcal{K}_{t}$. Since $R(\mathbf{K})$ is convex in $\mathbf{K}$, this means that $\mathcal{P}_{1}$ has an infinite number of non-convex constraints, which makes the problem very difficult to solve in its current form. Nevertheless, we show in the following lemma that the solution of $\mathcal{P}_{1}$ can be obtain by solving a convex optimization problem with a finite number of constraints.

Lemma 1. $\mathcal{P}_{1}$ is equivalent (in terms of optimal solution) to the following convex optimization problem.

$$
\begin{array}{cl}
\mathcal{P}_{2}: \quad \underset{t, \mathbf{K}}{\operatorname{minimize}} & t, \\
\text { subject to } & R(\mathbf{K}) \leq \bar{R}, \\
& \mathbf{K} \in \mathcal{K}_{t} .
\end{array}
$$

Proof: Please refer to Appendix A.

Problem $\mathcal{P}_{2}$ computes the IT by considering only the worstcase interference covariance matrix, i.e., that with the spatial structure that is the most detrimental for the primary receiver. Hence, if the rate constraint is guaranteed for the worst case, it will also be ensured for any other interference covariance matrix satisfying the IT constraint. Furthermore, we show in the following theorem that $\mathcal{P}_{2}$ admits a multilevel waterfilling solution.
Theorem 1. The worst-case interference covariance matrix, which is solution of $\mathcal{P}_{2}$, is given by

$$
\mathbf{K}^{\star}=\mathbf{\Gamma} \Lambda \boldsymbol{\Gamma}^{H}
$$

where $\Gamma$ is a unitary matrix containing the singular vectors of $\mathbf{H Q H}^{H}$, and $\Lambda$ is a positive diagonal matrix whose elements are given by a multilevel waterfilling as

$$
\lambda_{i}=\left[\sqrt{\phi_{i}\left(\frac{1}{4} \phi_{i}+\mu\right)}-\left(\frac{1}{2} \phi_{i}+\sigma^{2}\right)\right]^{+},
$$

where $\phi_{i}$ is the ith singular value of $\mathbf{H Q H}^{H}$ and $\mu$ such that the rate constraint is satisfied with equality.

Proof: Please refer to Appendix B.

In Theorem 1, the worst-case interference directions are derived, which correspond to the PU transmit eigenmodes. Also, the worst-case interference power along each direction is given in (9), whose form can be explained by looking at the derivative of the rate with respect to $\lambda_{i}$, which is given by

$$
\nabla_{\lambda_{i}} R\left(\mathbf{K}^{\star}\right)=\frac{1}{\log 2} \frac{\phi_{i}}{\left(\sigma^{2}+\lambda_{i}\right)\left(\sigma^{2}+\lambda_{i}+\phi_{i}\right)} .
$$

Plugging (9) into this expression, and assuming $\lambda_{i}>0$, we obtain

$$
\nabla_{\lambda_{i}} R\left(\mathbf{K}^{\star}\right)=\frac{1}{\mu \log 2},
$$

i.e., the derivative of the rate with respect to each non-null $\lambda_{i}$ is equal and inversely proportional to $\mu$. In other words, the optimal solution in (9) yields a point such that the variation of the rate of each PU signal mode with respect to its interference power is equal. Therefore, since (10) decreases with $\lambda_{i}$, any other interference power distribution with the same sum will increase the achievable rate.

In order to obtain the optimal value of $\mu$ in (9), we first notice that $\lambda_{i}$ increases monotonically with $\mu$ and, consequently, the achievable rate (right-hand side of (3)) decreases. Furthermore, $\mu$ can be bounded as follows.

Proposition 1. Let us assume that $\phi_{1} \geq \phi_{2} \geq \ldots \geq \phi_{N}$. The value of $\mu$ in (9), such that the rate constraint (6) holds with equality, can be bounded as

$\max \left\{\sigma^{2}\left(1+\frac{\sigma^{2}}{\phi_{1}}\right), \phi_{N} \frac{2^{\frac{\bar{R}}{N}}}{\left(2^{\frac{\bar{R}}{N}}-1\right)^{2}}\right\} \leq \mu \leq \phi_{1} \frac{2^{\frac{\bar{R}}{N}}}{\left(2^{\frac{\bar{R}}{N}}-1\right)^{2}}$.

Proof: Please refer to Appendix C.

These observations permit the application of onedimensional search methods, such as bisection or golden section, to obtain $\mu$.

\section{NUMERICAL EXAMPLES}

In this section we provide some numerical examples to illustrate our findings. We consider that each entry of the channel matrices are independent and identically distributed (i.i.d.) as $\mathcal{C N}(0,1)$. The transmit powers are set to 1 , thus 


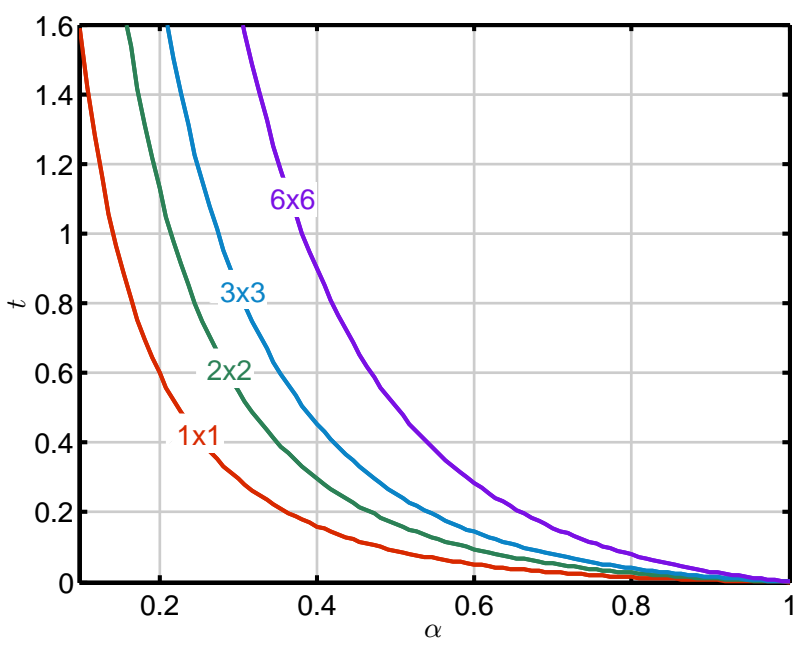

Fig. 1. Average IT for different systems as a function of the loading factor, $\alpha$.

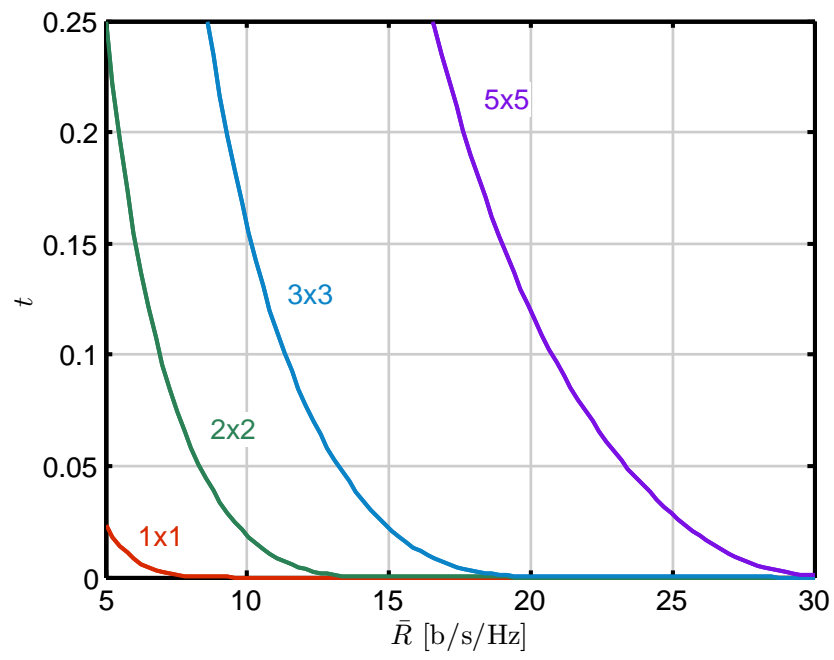

Fig. 2. Average IT for different systems as a function of $\bar{R}$.

the signal-to-noise ratio (SNR) is defined as $\log _{10} \frac{1}{\sigma^{2}}$, where $\sigma^{2}$ is the noise variance, and we fix it to $20 \mathrm{~dB}$. Without loss of generality, we assume that the PU performs the optimal strategy (in the absence of interference), i.e., singular value decomposition (SVD) of its direct channel and waterfilling power allocation [12]. Unless otherwise stated, we consider the rate constraint as a function of the point-to-point capacity (i.e., in the absence of interference) for each channel realization as $\bar{R}=\alpha R(\mathbf{0})$, where $\alpha \in[0,1]$ is the so-called loading factor. All results are averaged over 1000 independent channel realizations.

In Fig. 1 we plot the maximum IT, obtained according to Theorem 1, for different antenna configurations as a function of $\alpha$. Note that, for each value of $\alpha$, the rate requirement is higher for those systems whose capacity is also higher. We first observe that the curves behave similarly with $\alpha$. Second, systems with a higher number of antennas tolerate higher

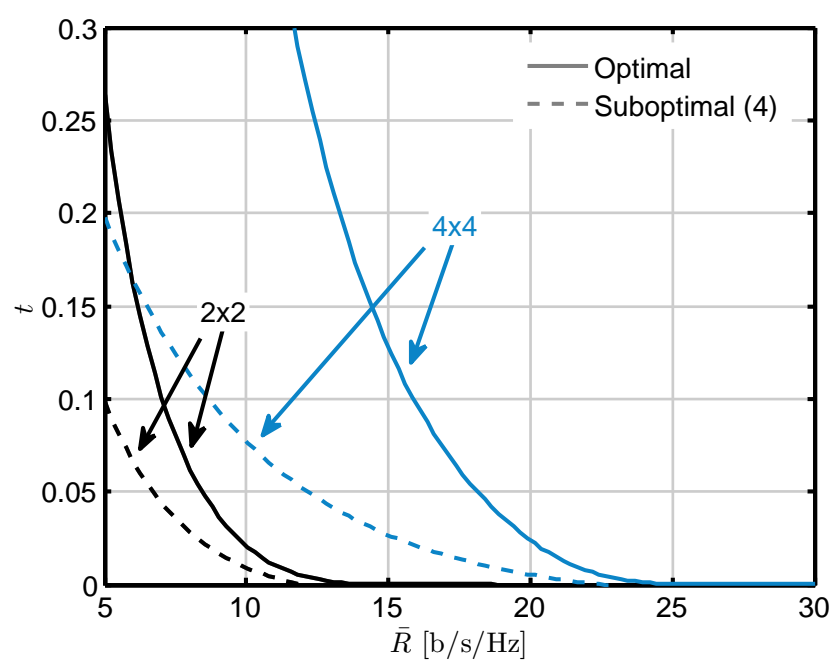

Fig. 3. Average IT for different systems as a function of $\bar{R}$. The suboptimal value (4) obtained in [11] is also depicted for comparison.

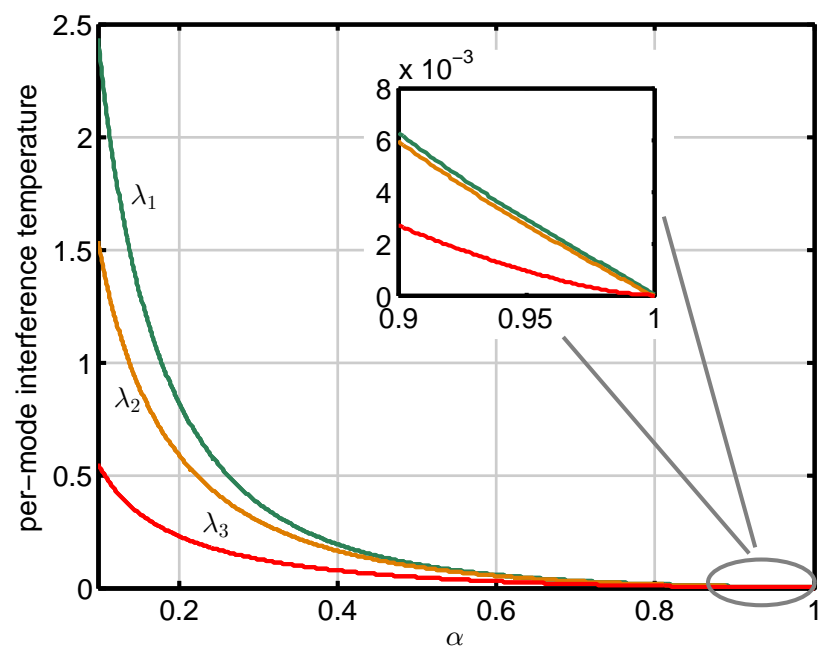

Fig. 4. Average IT per signal mode for a $3 \times 3 \mathrm{PU}$ as a function of $\alpha$.

amounts of interference in order to ensure the same percentage over their capacity. Also, the stringency of the IT becomes more notable when $\alpha$ goes from low to medium and high values (i.e., the slope of the curves is more prominent for low values of $\alpha$ ). Alternatively, we plot in Fig. 2 the optimal IT as a function of $\bar{R}$, when this is fixed independently of the capacity. Clearly, increasing the number of antennas, with $\bar{R}$ being fixed, provides a huge increase of the IT, as the additional antennas are solely used to relax the IT limit. In Fig. 3, the maximum IT obtained from Theorem 1 is shown for $2 \times 2$ and $4 \times 4$ MIMO systems as a function of $\bar{R}$, and compared with the bound given in (4). As shown in the figure, not using the maximum IT may yield pessimistic or too conservative results, specially when the PU is not highly loaded.

Finally, we depict in Fig. 4 the average IT per signal mode, $\lambda_{i}$, as a function of $\alpha$. Interestingly, all of them are non-null in almost the whole $\alpha$ regime. 


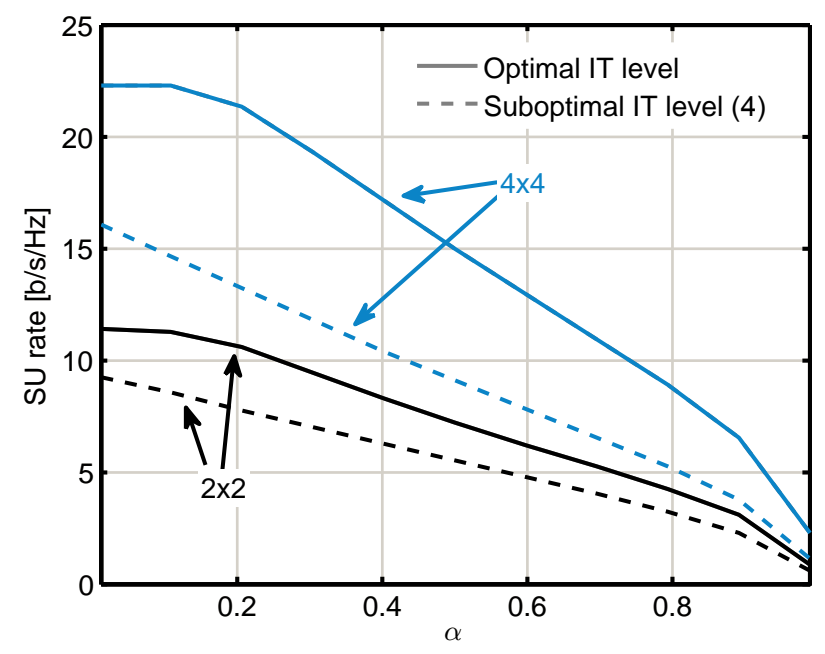

Fig. 5. Average rate of a SU constrained by the IT as a function of $\alpha$.

\section{A. Secondary rate maximization}

We finally illustrate the usefulness of our result with a practical example. Consider a single SU coexisting with a PU that has a rate constraint as in (3). For simplicity, and without loss of generality, let us assume that there is no interfering link from the primary transmitter to the secondary receiver. Consider also that the number of transmit and receive antennas is equal for both PU and SU and is given by $N$. The secondary transmit covariance matrix that maximizes the SU rate can be found by solving the following convex optimization problem.

$$
\begin{array}{cl}
\mathcal{P}_{3}: \quad \underset{\mathbf{P}}{\operatorname{maximize}} & \log _{2}\left|\mathbf{I}+\frac{1}{\sigma^{2}} \mathbf{F P F}^{H}\right|, \\
\text { subject to } & \operatorname{Tr}(\mathbf{P}) \leq 1, \\
& \mathbf{P} \succeq \mathbf{0}, \\
& \operatorname{Tr}\left(\mathbf{G P G}{ }^{H}\right) \leq t,
\end{array}
$$

where $\mathbf{F} \in \mathbb{C}^{N \times N}$ and $\mathbf{G} \in \mathbb{C}^{N \times N}$ are SU-SU and SU-PU channels, and $\mathbf{P} \in \mathbb{C}^{N \times N}$ is the transmit covariance matrix of the SU. Notice that the last constraint in $\mathcal{P}_{3}$ is the IT constraint to ensure that the PU meets its minimum rate requirement. Since this problem is convex, it can be efficiently solved by standard numerical methods [13]. For this scenario, we plot in Fig. 5 the average achievable rate of the SU as a function of $\alpha$, for $N=2$ and $N=4$ antennas. We observe that there is a significant rate difference between using the optimal IT limit and the suboptimal threshold given by (4), which becomes more prominent as the number of antennas increases.

\section{CONCLUSION}

In this paper we have addressed the problem of obtaining the IT limit for a MIMO PU that has a rate constraint. As opposed to the single-antenna case, the mapping between interference power and achievable rate is not unique, since the spatial structure of the interference also affects the rate of the PU. Hence, the IT limit must be chosen in such a way that the rate is ensured independently of the spatial signature of the interference. To this end, we have shown that it is sufficient to obtain the worst-case interference covariance matrix, whose structure has been also derived and expressed in closed-form. These results allow to efficiently compute the optimal IT limit, which permits a better analysis of underlay CR networks and a correct assessment of alternative approaches to IT.

\section{ACKNOWLEDGEMENT}

C. Lameiro and I. Santamaría have received funding from the Spanish Government (MICINN) under projects CONSOLIDER-INGENIO 2010 CSD2008-00010 (COMONSENS), TEC2013-47141-C4-3-R (RACHEL) and FPU Grant AP2010-2189. W. Utschick receives financial support from the Deutsche Forschungsgemeinschaft (DFG) under the grant Ut36/15-1.

\section{APPENDIX A}

\section{PROOF OF LEMMA 1}

Let $t_{1}^{\star}$ and $t_{2}^{\star}$ be the optimal solution of $\mathcal{P}_{1}$ and $\mathcal{P}_{2}$, respectively. Clearly $t_{2}^{\star} \leq t_{1}^{\star}$, which implies $\mathcal{K}_{t_{2}^{\star}} \subseteq \mathcal{K}_{t_{1}^{\star}}$. Let $\mathbf{K}_{1} \in \mathcal{K}_{t_{1}^{\star}}$ be such that $\bar{R}\left(\mathbf{K}_{1}\right)=\bar{R}$; and $\mathbf{K}_{2} \in \mathcal{K}_{t_{2}^{\star}}$ such that $R\left(\mathbf{K}_{2}\right)=\bar{R}$. Now take $\tilde{\mathbf{K}}=\frac{t_{1}^{\star}}{t_{2}^{\star}} \mathbf{K}_{2}$. It is clear that $\tilde{\mathbf{K}} \in \mathcal{K}_{t_{1}^{\star}}$ and $\tilde{\mathbf{K}} \succeq \mathbf{K}_{2}$. As $R(\mathbf{A}) \geq R(\mathbf{B})$ for $\mathbf{A} \preceq \mathbf{B}$, we obtain $R(\tilde{\mathbf{K}}) \leq R\left(\mathbf{K}_{2}\right)=\bar{R}$. Since $R(\mathbf{K}) \geq \bar{R}$ for all $\mathbf{K} \in \mathcal{K}_{t_{1}^{\star}}$, $\tilde{\mathbf{K}}=\mathbf{K}_{2}$ must hold, implying $t_{1}^{\star}=t_{2}^{\star}$, which concludes the proof.

\section{APPENDIX B}

\section{PROOF OF THEOREM 1}

Let $\mathbf{H Q H} \mathbf{H}^{H}=\boldsymbol{\Xi} \boldsymbol{\Phi} \boldsymbol{\Xi}^{H}$ and $\mathbf{K}=\boldsymbol{\Gamma} \boldsymbol{\Lambda} \boldsymbol{\Gamma}^{H}$ be the singular value decomposition (SVD). Since the directions of $\mathbf{K}$ only affect the rate constraint, it is clear that $\Gamma=\Xi$ must hold for the optimal solution of $\mathcal{P}_{2}$. Taking this into account, $\mathcal{P}_{2}$ can be reduced to

$$
\begin{aligned}
\tilde{\mathcal{P}}_{2}: \quad \underset{\left\{\lambda_{i}\right\}_{i=1}^{N}}{\operatorname{minimize}} & \sum_{i=1}^{N} \lambda_{i}, \\
\text { subject to } & \sum_{i=1}^{N} \log _{2}\left(1+\frac{\phi_{i}}{\sigma^{2}+\lambda_{i}}\right) \leq \bar{R}, \\
& \lambda_{i} \geq 0, i=1, \ldots, N,
\end{aligned}
$$

Since this problem is convex and satisfies the Slater's condition [13], its optimal solution can be found by solving the dual problem. To this end, let us consider the Lagrangian of $\tilde{\mathcal{P}}_{2}$, given by

$$
\begin{aligned}
& \mathscr{L}\left(\left\{\lambda_{i}\right\}_{i=1}^{N},\left\{\nu_{i}\right\}_{i=1}^{N}, \tilde{\mu}\right)=\sum_{i=1}^{N} \lambda_{i}+ \\
& \tilde{\mu}\left[\sum_{i=1}^{N} \log _{2}\left(1+\frac{\phi_{i}}{\sigma^{2}+\lambda_{i}}\right)-\bar{R}\right]-\sum_{i=1}^{N} \nu_{i} \lambda_{i},
\end{aligned}
$$

where $\tilde{\mu}$ and $\nu_{i}(i=1, \ldots, N)$ are the Lagrange multipliers of (13) and (14), respectively. Then, the Karush-Kahn-Tucker 
(KKT) conditions for this problem read

$$
\begin{aligned}
& \sum_{i=1}^{N} \log _{2}\left(1+\frac{\phi_{i}}{\sigma^{2}+\lambda_{i}}\right) \leq \bar{R}, \lambda_{i} \geq 0, i=1, \ldots, N \\
& \tilde{\mu} \geq 0, \nu_{i} \geq 0, i=1, \ldots, N \\
& \tilde{\mu}\left[\sum_{i=1}^{N} \log _{2}\left(1+\frac{\phi_{i}}{\sigma^{2}+\lambda_{i}}\right)-\bar{R}\right]=0 \\
& \nu_{i} \lambda_{i}=0, i=1, \ldots, N, \\
& \nabla_{\lambda_{i}} \mathscr{L}\left(\left\{\lambda_{i}\right\}_{i=1}^{N},\left\{\nu_{i}\right\}_{i=1}^{N}, \tilde{\mu}\right)=0, i=1, \ldots, N
\end{aligned}
$$

As strong duality holds, the optimal $\lambda_{i}, \tilde{\mu}$ and $\nu_{i}$ must satisfy the foregoing conditions. By evaluating the last one we obtain

$$
\begin{aligned}
& \nabla_{\lambda_{i}} \mathscr{L}\left(\left\{\lambda_{i}\right\}_{i=1}^{N},\left\{\nu_{i}\right\}_{i=1}^{N}, \tilde{\mu}\right)=1- \\
& \mu \frac{\phi_{i}}{\left(\sigma^{2}+\lambda_{i}\right)\left(\sigma^{2}+\lambda_{i}+\phi_{i}\right)}-\nu_{i}=0 \\
& \quad \Rightarrow \lambda_{i}=\sqrt{\phi_{i}\left(\frac{1}{4} \phi_{i}+\frac{\mu}{1-\nu_{i}}\right)}-\left(\frac{1}{2} \phi_{i}+\sigma^{2}\right),
\end{aligned}
$$

where $\mu=\frac{\tilde{\mu}}{\log 2}$. Due to condition (19), we have that $\nu_{i}=$ $0 \Leftrightarrow \lambda_{i}>0$ and $\nu_{i}>0 \Leftrightarrow \lambda_{i}=0$, which, combined with (21), yields (9) and concludes the proof.

\section{APPENDIX C PROOF OF PROPOSITION 1}

First, it can be easily checked that $\phi_{1} \geq \phi_{2} \geq \ldots \geq \phi_{N}$ implies $\lambda_{1} \geq \lambda_{2} \geq \ldots \geq \lambda_{N}$. Now consider the following lower bound on the achievable rate

$$
\sum_{i=1}^{N} \log _{2}\left(1+\frac{\phi_{i}}{\sigma^{2}+\lambda_{i}}\right) \geq N \log _{2}\left(1+\frac{\phi_{N}}{\sigma^{2}+\lambda_{N}}\right) .
$$

In the optimal point, the achievable rate is equal to $\bar{R}$, hence the lower bound in the right-hand side of (22) satisfies

$$
N \log _{2}\left(1+\frac{\phi_{N}}{\sigma^{2}+\lambda_{N}}\right) \leq \bar{R} \Rightarrow \lambda_{N} \geq \frac{\phi_{N}}{2^{\frac{\bar{R}}{N}}-1}-\sigma^{2} .
$$

Combining the right-hand side of this expression with (9) yields

$$
\begin{aligned}
& \sqrt{\phi_{N}\left(\frac{1}{4} \phi_{N}+\mu\right)}-\frac{1}{2} \phi_{1}-\sigma^{2} \geq \frac{\phi_{N}}{2^{\frac{\bar{R}}{N}}-1}-\sigma^{2} \\
& \Rightarrow \mu \geq \phi_{N} \frac{2^{\frac{\bar{R}}{N}}}{\left(2^{\frac{\bar{R}}{N}}-1\right)^{2}} .
\end{aligned}
$$

On the other hand, we also have $\lambda_{i} \geq 0$. Thus, taking $\lambda_{1} \geq 0$ we obtain

$$
\begin{aligned}
\lambda_{1} \geq 0 & \Rightarrow \sqrt{\phi_{1}\left(\frac{1}{4} \phi_{1}+\mu\right)} \geq \frac{1}{2} \phi_{1}+\sigma^{2} \\
& \Rightarrow \mu \geq \sigma^{2}\left(1+\frac{\sigma^{2}}{\phi_{1}}\right)
\end{aligned}
$$

The lower bound on $\mu$ is then obtained combining (24) with (25).

Similarly, the achievable rate can be upper-bounded as

$$
\sum_{i=1}^{N} \log _{2}\left(1+\frac{\phi_{i}}{\sigma^{2}+\lambda_{i}}\right) \leq N \log _{2}\left(1+\frac{\phi_{1}}{\sigma^{2}+\lambda_{1}}\right) .
$$

Again, since at the optimal point the achievable rate is equal to $\bar{R}$, we have

$$
N \log _{2}\left(1+\frac{\phi_{1}}{\sigma^{2}+\lambda_{1}}\right) \geq \bar{R} \Rightarrow \lambda_{1} \leq \frac{\phi_{1}}{2^{\frac{\bar{R}}{N}}-1}-\sigma^{2},
$$

which, combined with (9), yields

$$
\begin{aligned}
& \sqrt{\phi_{1}\left(\frac{1}{4} \phi_{1}+\mu\right)}-\frac{1}{2} \phi_{1}-\sigma^{2} \leq \frac{\phi_{1}}{2^{\frac{\bar{R}}{N}}-1}-\sigma^{2} \\
& \Rightarrow \mu \leq \phi_{1} \frac{2^{\frac{\bar{R}}{N}}}{\left(2^{\frac{\bar{R}}{N}}-1\right)^{2}},
\end{aligned}
$$

and concludes the proof.

\section{REFERENCES}

[1] J. Mitola and G. Maguire, "Cognitive Radio: Making Software Radios More Personal," IEEE Personal Communications, vol. 6, no. 4, pp. 13$18,1999$.

[2] S. Haykin, "Cognitive Radio: Brain-Empowered Wireless Communications," IEEE Journal on Selected Areas in Communications, vol. 23, no. 2, pp. 201-220, Feb. 2005.

[3] A. Goldsmith, S. Jafar, I. Maric, and S. Srinivasa, "Breaking Spectrum Gridlock With Cognitive Radios: An Information Theoretic Perspective," Proceedings of the IEEE, vol. 97, no. 5, pp. 894-914, May 2009.

[4] Y. Yang, G. Scutari, P. Song, and D. P. Palomar, "Robust MIMO Cognitive Radio Systems Under Interference Temperature Constraints," IEEE Journal on Selected Areas in Communications, vol. 31, no. 11, pp. 2465-2482, Nov. 2013.

[5] G. Scutari and D. Palomar, "MIMO Cognitive Radio: A Game Theoretical Approach," IEEE Transactions on Signal Processing, vol. 58, no. 2, pp. 761-780, Feb. 2010.

[6] R. Zhang, Y.-c. Liang, and S. Cui, "Dynamic Resource Allocation in Cognitive Radio Networks," IEEE Signal Processing Magazine, vol. 27, no. 3, pp. 102-114, May 2010.

[7] R. Zhang and Y.-c. Liang, "Exploiting Multi-Antennas for Opportunistic Spectrum Sharing in Cognitive Radio Networks," IEEE Journal of Selected Topics in Signal Processing, vol. 2, no. 1, pp. 88-102, Feb. 2008.

[8] L. Zhang, Y.-c. Liang, and Y. Xin, "Joint Beamforming and Power Allocation for Multiple Access Channels in Cognitive Radio Networks," IEEE Journal on Selected Areas in Communications, vol. 26, no. 1, pp. 38-51, Jan. 2008.

[9] S.-J. Kim and G. B. Giannakis, "Optimal Resource Allocation for MIMO Ad Hoc Cognitive Radio Networks," IEEE Transactions on Information Theory, vol. 57, no. 5, pp. 3117-3131, May 2011.

[10] T. M. Cover and J. A. Thomas, Elements of Information Theory, ser. Wiley Series in Telecommunications. New York, USA: John Wiley \& Sons, Inc., 1991

[11] K. Cumanan, R. Zhang, and S. Lambotharan, "A New Design Paradigm for MIMO Cognitive Radio with Primary User Rate Constraint," IEEE Communications Letters, vol. 16, no. 5, pp. 706-709, May 2012.

[12] I. E. Telatar, "Capacity of multi-antenna gaussian channels," European Transactions on Telecommunications, vol. 10, no. 6, pp. 585-595, November-December 1999.

[13] S. Boyd and L. Vandenberghe, Convex Optimization. Cambridge University Press, 2004. 